\title{
Plasma torch for supersonic plasma spray at atmospheric pressure
}

\author{
F.R. Caliari ${ }^{\mathrm{a}, \mathrm{b}, *}$, F.S. Miranda ${ }^{\mathrm{b}}$, D.A.P. Reis ${ }^{\mathrm{a}}$, G.P. Filho ${ }^{\mathrm{b}}$, L.I. Charakhovski ${ }^{\mathrm{c}}$, A. Essiptchouk ${ }^{\mathrm{d}}$ \\ a Instituto de Ciência e Tecnologia, Universidade Federal de São Paulo - UNIFESP, São José dos Campos 12231-280, SP, Brazil

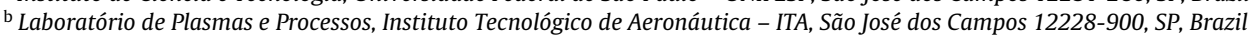 \\ ${ }^{\mathrm{c}}$ Luikov Heat and Mass Transfer Institute, HMTI - National Academy of Sciences of Belarus, Minsk 220072, Belarus \\ dinstituto de Ciência e Tecnologia, Univ. Estadual Paulista - UNESP, São José dos Campos 12247-004, SP, Brazil
}

\section{A R T I C L E I N F O}

\section{Article history:}

Received 28 October 2015

Received in revised form 17 March 2016

Accepted 18 June 2016

Available online 23 June 2016

Keywords:

Atmospheric plasma spray

Plasma torch

Supersonic plasma spray

Axial injection

In-flight particle characteristics

\begin{abstract}
A B S T R A C T
This work presents a plasma torch able to operate at supersonic regime with axial injection of feedstock. In contrast to commonly used linear scheme, the principal axis of the plasma torch is perpendicular to feedstock injection direction, which is aligned with coming out plasma jet. The plasma torch has slightly ascending current voltage characteristics and fixed arc length. Electrical, thermal and kinetic characteristics outlined from comparison with conventional linear plasma spray torches are intermediate between APS, HVOF and VPS. The plasma torch developed in this work has an elevated arc voltage (370 V) and low arc current $(100 \mathrm{~A})$, which contribute to increase the electrode life and decrease the arc voltage relative fluctuation (10\%). According to in-flight particle monitoring the CoNiCrAlY particles were sprayed at $500 \mathrm{~m} / \mathrm{s}$ and temperature of $2400^{\circ} \mathrm{C}$, whereas the $7 \% \mathrm{YSZ}$ at $491-683 \mathrm{~m} / \mathrm{s}$ and $2535-2636^{\circ} \mathrm{C}$.
\end{abstract}

(c) 2016 Elsevier B.V. All rights reserved.

\section{Introduction}

Thermal spray embodies a family of processes where a layer of material (metallic, ceramic and/or polymer) is applied on a substrate in order to get protection against oxidation, corrosion, abrasion, thermal loads, high temperature fatigue and creep. Hermanek (2001) defined the thermal spraying as "a group of coating processes in which finely divided metallic or non-metallic materials are deposited in a molten or semi-molten condition to form a coating". Thus the coatings are produced when the particles are heated and deformed at impact with the substrate, which happens if they are not only softened but have sufficient kinetic energy. The particles may be in the form of a powder, solution or suspension, and from here on it shall be called generically as feedstock.

The thermal spraying encloses several processes that use the thermal energy generated chemically (by combustion) or electrically (mainly by electric arc discharge) to soften and/or melt and accelerate the feedstock at high velocities from few tens to thousand meters per second.

Fauchais and Montavon, 2007 highlighted that among the thermal spraying processes, the atmospheric plasma spray (APS) is the

\footnotetext{
* Corresponding author.

E-mail address: felipercaliari@yahoo.com.br (F.R. Caliari).
}

technique most commonly used due to its versatility and costefficiency. The high temperature of the plasma jet is suitable, but is not limited, to materials with high melting point like ceramics and refractory materials. The process usually is accomplished in the open-air environment, but to improve the coating quality a controlled atmosphere chamber (LPPS - low pressure plasma spray or VPS - vacuum plasma spray) may be used.

As described by Pawlowski (2008) thermal energy converted from electrical determines the temperature of the flame (or jet), however, besides the efficiency of fusion of the particles, the adhesion of droplets to the substrate and porosity depends also of the plasma velocity. The conventional plasma spray (APS) has higher thermal loads and relatively low particles velocities when compared to High Velocity Oxygen Fuel (HVOF), Detonation Gun (D-Gun) and Vacuum Plasma Spray process (VPS).

Most conventional torches for plasma spraying were developed in decade 60 . With the aim to improve the plasma spraying technique, a considerable number of plasma torches types, using different physical principles, have been developed in the past (see for example Zhukov and Zasypkin, 2007). Most of them used linear-circuit plasma generators where the electrodes (cathode and anode) are arranged axially. The cathode, normally, is the inner electrode and output nozzle is the anode.

A discharge chamber of typical plasma torch consists of one (usually tungsten rod) surrounded by a concentric hollow anode, which acts as output nozzle, as shown by Zhukov and Zasypkin 
(2007). An electric arc ignited between the electrodes and the gas flow blow-out a high enthalpy plasma jet. The feedstock is injected (radially or axially) into the plasma jet where is melted and accelerated in the substrate direction. The advantage of this type of plasma torch is its simplicity, small number of parts and extensive set of well-known operating parameters. The arc is practically immobile on the cathode surface, while it has axial and radial motions on the anode surface, which result in oscillation of the arc voltage and, hence, in the enthalpy of the plasma jet, as described by Nogues et al. (2008).

Fauchais et al. (2013a,b) pointed out that longitudinally blown arc possess best energetic characteristics, but it is commonly associated with radial injection of feedstock, which stimulates elevated dispersion and spatial segregation by grain size distribution. Chyou and Pfender (1989) reinforced that the plasma torches provide high temperatures but, to be an effective heat source, its interaction with the feedstock depends of various parameters. The estimation of inflight particle properties can be a difficult task because one must set correctly the heat transfer boundary conditions, which depends on particle morphology and size, Biot number, injection method, among other parameters, as observed by Fauchais and Montavon, 2007. Depending on the injection method used, the relationship between the particle and plasma jet momentum will be affected, along with the trajectory and the particle residence time. Besides, acceleration of particles at low-density plasma jet is not as effective as at high pressure and high density.

Vardelle et al. (1994) developed a plasma torch with axial injection considering the path of the particle through the cathode, which in principle allows a close contact with the electric arc, but reduces the lifetime of the cathode due to erosion. Mohanty et al. (2010) studied a linear plasma torch with the particle injection through the cathode, and the results showed that the introduction of particles did not affected overall arc behaviour, although an elevated wear of the cathode was observed. Vardelle et al. (2015) pointed out that the advantages provided by the axial injection method are the maximization of particles injected, uniform heating and elevated deposition efficiency. According to Fauchais et al. (2011) the Mettech Axial III plasma torch is composed of three cathodes/anodes. The plasma jets, generated in separated channels, join together in a single outlet, where the feedstock is injected. This configuration provides high residence time.

Papyrin et al. (2006) and Van Steenkiste et al. (1999) affirmed that supersonic or hypersonic plasma jets permit to obtain dense spray coatings, similar to kinetic spray. One can hypothesize that application of axial injection and acceleration of particles combined with supersonic plasma jet would make it possible to fill the gap between dense but low melting coating materials obtained by low-temperature kinetic spray and, refractory coatings obtained by traditional plasma spray with substantially higher porosity. In this work, a plasma torch working with axial injection and supersonic regime, along with the in-flight particles analysis of metallic and ceramic powders plasma sprayed is presented.

\section{Materials and methods}

\subsection{Feedstock materials}

In this work the metallic powder Amperit 415.001, CoNiCrAlY, gas atomized, from HC Stark, and the ceramic powder Amperit 825.000, 7\%YSZ, fused and crushed, from HC Stark, were studied. Before each test, the powders were dried for $1 \mathrm{~h}$ at $100^{\circ} \mathrm{C}$. Chemical composition and the grain size distribution of powders are illustrated on Table 1.
Table 1

Chemical composition and particle size of powders.

\begin{tabular}{lllllllllll}
\hline \multirow{2}{*}{ Powder } & \multicolumn{3}{l}{ Composition $(w t . \%)$} & \multicolumn{1}{c}{ Particle size distribution $(\mu \mathrm{m})$} \\
\cline { 2 - 7 } & $\mathrm{Co}$ & $\mathrm{Ni}$ & $\mathrm{Cr}$ & $\mathrm{Al}$ & $\mathrm{Y}$ & $\mathrm{ZrO}_{2}$ & $\mathrm{Y}_{2} \mathrm{O}_{3}$ \\
\hline CoNiCrAlY & Bal & 32 & 21 & 8 & 0.5 & - & - & $22-45$ \\
$7 \% \mathrm{YSZ}$ & - & - & - & - & - & Bal. & 7 & $5-22$ \\
\hline
\end{tabular}

\subsection{In-flight experimental analysis}

The kinetic and thermal characteristics of HVPS process is herein ascertained by means of in-flight particle velocity and temperature. The in-flight particle state was measured using a DPV-2000, 2009 Premium (Tecnar, St. Bruno, QC, Canada, 2009), which uses an infrared pyrometer system to collect particle individual data on the plasma stream. For all in-flight particle measurements performed with DPV-2000, the auto-center procedure was performed in order to set the position within the plasma plume with maximum data acquisition. For each test at least 3000 particles data were collected, to guarantee a reasonable level of statistical significance.

\subsection{Plasma spray experimental set-up}

This work presents a two-chamber design of plasma torch for high velocity plasma spraying, briefly described by Caliari et al. (2015). Development of this plasma torches is motivated by the request to improve the arc stability and to increase the range of powders to be used and quality of sprayed coating surface. Thermal and kinetic parameters from the plasma torch have been described by means of in-flight particle properties. The plasma torch is designed to work at sub- and supersonic regimes. The principal difference from other constructions is that the discharge chamber axis is perpendicular to powder injection that is aligned with coming out plasma jet. The schematic diagram of plasma torch for High Velocity Plasma Spray (HVPS) proposed in this work is shown in Fig. 1. The mixing chamber is placed between two tubular copper electrodes. The swirled gas enters the electrodes (where a reversed vortex flow is formed) and the mixing chamber (with direct vortex), stabilizing the electric arc at the principal axis of the plasma torch. The nozzle is arranged in mixing chamber perpendicularly and eccentrically from the principal axis according to the direction of vortex rotation. Therefore, the arc plasma enters the nozzle with no swirling. Feedstock is injected through nipple coaxially to the nozzle by the flow of additional transporting gas. Elevated temperature of the mixing chamber walls, as well as optimization of particle injection, is necessary for preventing the material adhesion on the walls and possible clogging of the nozzle. An additional magnetic field can be used for the stabilization of the anode axial position. In presented work, in order to fix the arc length and, thus, decrease the arc voltage and enthalpy fluctuations, a plain anode with a protective argon atmosphere was used.

This plasma torch is designed for up to $50 \mathrm{~kW}$ of power input and uses nitrogen as plasma forming gas. The power supply was arranged on the basis of six welding transformers with magnetic shunts controlling impedance and external characteristics. The transformers have been connected in series-parallel groups and, together with the standard three-phase rectifier, the DC power supply with an off-load voltage of $675 \mathrm{~V}$ and descending $\mathrm{V}(\mathrm{I})$ characteristics turned out.

The plasma torch is ignited by a high frequency/voltage, which promotes simultaneous breakdown of the gaps formed between mixing chamber and electrodes (cathode and anode). Started main arc is blowing out and stabilized on torch axis by vortex.

A preliminary modelling of internal cold flow of HVPS is presented on Fig. 2. The internal cold flow inside of plasma torch chamber was preliminary simulated with SOLIDWORKS Flow Sim- 

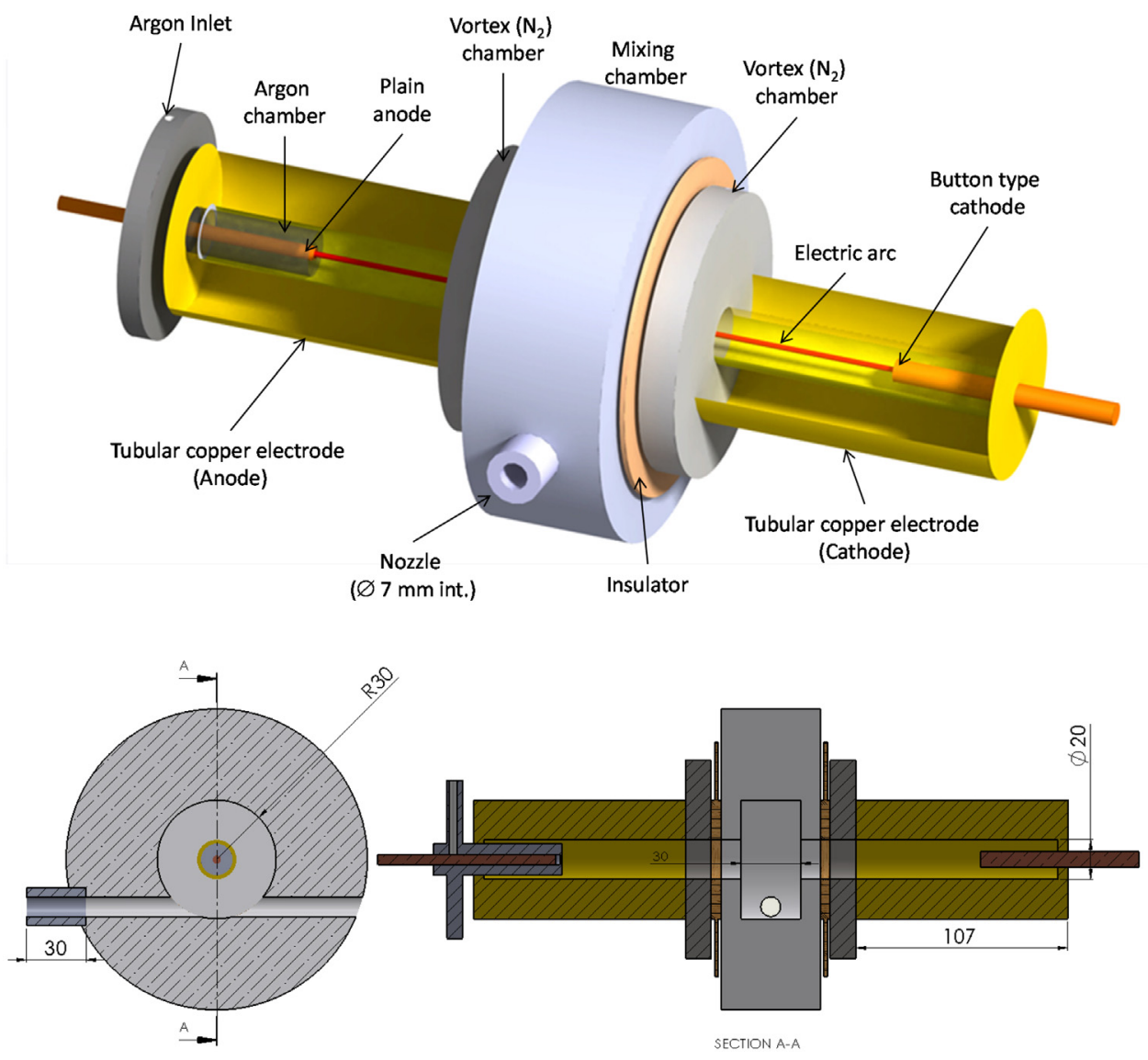

Fig. 1. Schematic diagram of plasma torch for high velocity plasma spraying.

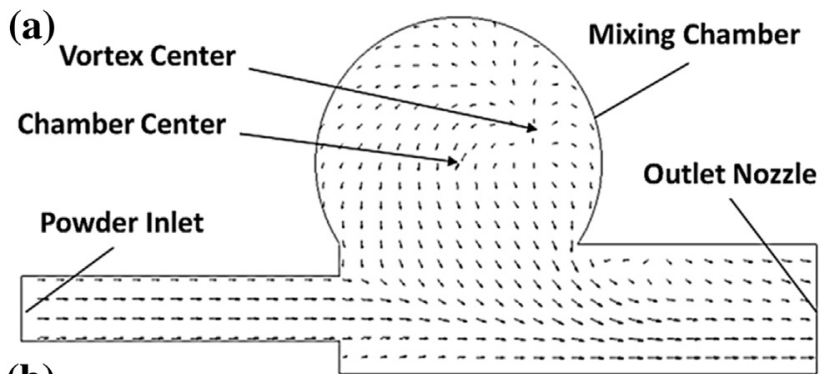

(b)

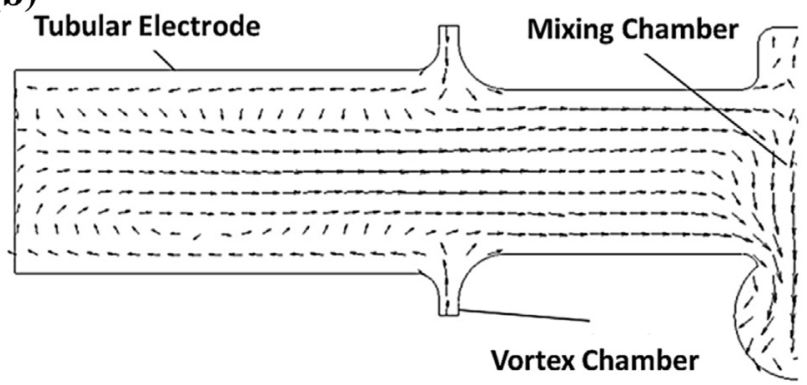

Fig. 2. Velocity flow pattern in (a) transversal section of mixing chamber (b) and axial section of electrode.

ulation package that used Computational Fluid Dynamics (CFD) analysis. Fluid motion is modelled by using the Navier-Stokes equations, which describe, in non-stationary formulation, the laws of conservation of mass, momentum and energy of the medium. A 3D model of inner cavity of plasma torch was studied at initial pressure $1.01325 \mathrm{MPa}$ and temperature $20^{\circ} \mathrm{C}$. The inner walls of the chamber were defined as adiabatic with zero roughness. The flow type laminar and turbulent was assumed. For this, the transport equation of turbulent kinetic energy and its dissipation within k- $\varepsilon$ model of turbulence was taken into account. The turbulence energy of $1 \mathrm{~J} / \mathrm{kg}$ and turbulence dissipation of $1 \mathrm{~W} / \mathrm{kg}$ was assumed in simulation. Boundary condition of Nitrogen gas flow rate $3 \mathrm{~g} / \mathrm{s}$ and outlet environment pressure of $1.01325 \mathrm{MPa}$ were placed. From the gas velocity pattern, a good vortex is formed in the inner tubular electrode channel that must stabilize well the arc on the centre of the axis. In the mixing chamber, a vortex is off-centred and may induce augment in the arc oscillations.

In order to achieve a predetermined Mach number at the exit section of a supersonic nozzle, a cross-sectional area must be suitably chosen in accordance with gas flow rate and, moreover, it is necessary to have an adequate supply pressure in the discharge chamber of plasma torch. On the other hand, the exhaust velocity of a gas in a supersonic nozzle depends only on the stagnation temperature $T_{0}$. A change of the total pressure $p_{0}$ in the discharge chamber does not affect the velocity, since a local pressure $p$ is changed proportionally and their ratio $p / p_{0}$ remains unchanged, as well as the ratio of temperatures $\left(T / T_{0}\right)$,

$\frac{p}{p_{0}}=\left(\frac{T}{T_{0}}\right)^{\frac{\gamma}{\gamma-1}}$

Preliminary calculations showed that at moderate, for a nitrogen plasma gas, temperature $1720-2720^{\circ} \mathrm{C}$ and plenum pressure $0.5 \mathrm{MPa}$, a velocity of nitrogen plasma achieves $1200-1600 \mathrm{~m} / \mathrm{s}$. Axial injection of particles at subsonic part of jet at the direction of gas flow, the same manner as used in kinetic spray, must allow a more effective acceleration. Expected parameters of HVPS pro- 


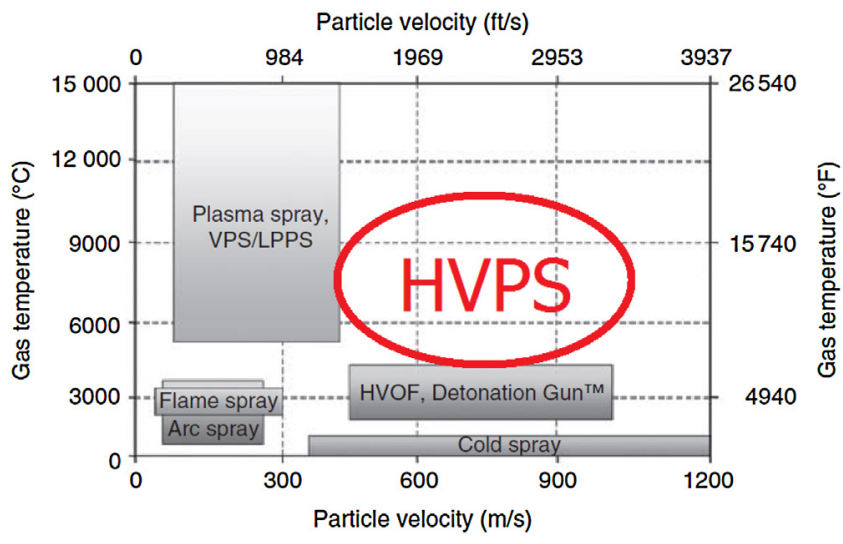

Fig. 3. Gas temperature versus particle velocity of thermal spray processes. APSAtmospheric Plasma Spray, LPPS-Low Pressure Plasma Spray, VPS-Vacuum Plasma Spray. HVOF-High Velocity Oxygen Fuel, HVPS-High Velocity Plasma Spray [This picture was published in the book: The cold spray materials deposition process Fundamentals and applications, M. F. Smith, Comparing cold spray with thermal spray coating technologies, pp. 43-61, Copyright Elsevier, 2007].

cess in comparison with traditional thermal spraying processes are shown in Fig. 3.

During the plasma spray experiments a 4MP dual powder feeder adapted to operate at high pressures was used. Table 2 describes the set of parameters of HVPS process, as well as comparison to conventional APS parameters.

The average plasma jet enthalpy $(\Delta \mathrm{h})$ has been calculated from the plasma torch parameters. The total input power of electric arc discharge, $\mathrm{P}_{\text {total }}, \mathrm{W}$, is calculated as follow:

$P_{\text {total }}=U I$,

where, $\mathrm{U}$ is the arc voltage, $\mathrm{V}$, and I is the arc current, A. The dissipated energy $\left(\mathrm{P}_{\mathrm{AB}}\right)$ in plasma torch cooling system was estimated by measuring the water-cooling mass flow rate, $\mathrm{G}_{\mathrm{H} 2 \mathrm{O}}, \mathrm{kg} / \mathrm{s}$, and the temperature gradient $\Delta \mathrm{T}, \mathrm{K}$, of the cooling water

$P_{A B}=G_{\mathrm{H}_{2} \mathrm{O}} c_{p_{\mathrm{H} 2 \mathrm{O}}} \Delta T$,

where $c_{p_{\mathrm{H} 2 \mathrm{O}}}$ is the specific heat capacity of water. Thus the thermal efficiency, $\eta$, of the plasma torch is:

$\eta=\frac{P_{\text {total }}-P_{A B}}{P_{\text {total }}}$.

The bulk enthalpy variation of the plasma jet, $\Delta \mathrm{h}, \mathrm{kJ} / \mathrm{kg}$, is obtained by the ratio between the net power and total mass flow rate of gas (nitrogen) injected into the plasma torch, i.e.

$\Delta h=\frac{\eta P_{\text {total }}}{G_{N 2}+G_{\text {spray }}}$,

Here, $G_{N 2}$ and $G_{\text {spray }}$ are the mass flow rate of the working gas and the carrier gas, respectively, $\mathrm{kg} / \mathrm{s}$. In this calculation the sprayed powder influence on $\Delta h$ was neglected.

\section{Results and discussion}

A supersonic plasma jet produced during HVPS is shown in Fig. 4. When the discharge chamber pressure exceeds the designed one, the exit pressure of plasma jet is higher than the external pressure and this flow is called under expanded. This overpressure is consumed due to the increase of the plasma jet velocity. Acceleration of the supersonic flow requires an increase in the jet cross section, thus the plasma jet forms in the space an expanding "supersonic nozzle". In certain moment, with the wider section of the jet, the pressure established is lower than the atmospheric one. After that, the jet begins to contract, because the pressure should attain

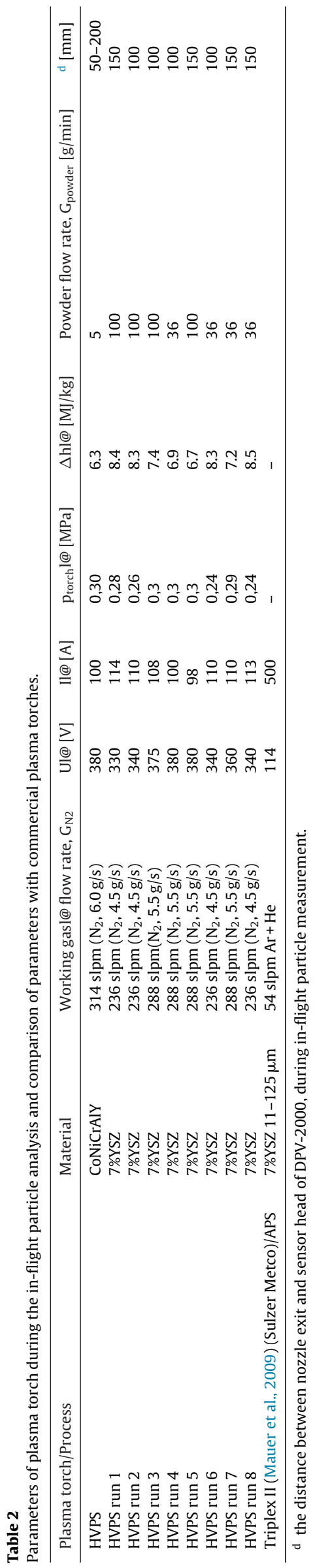




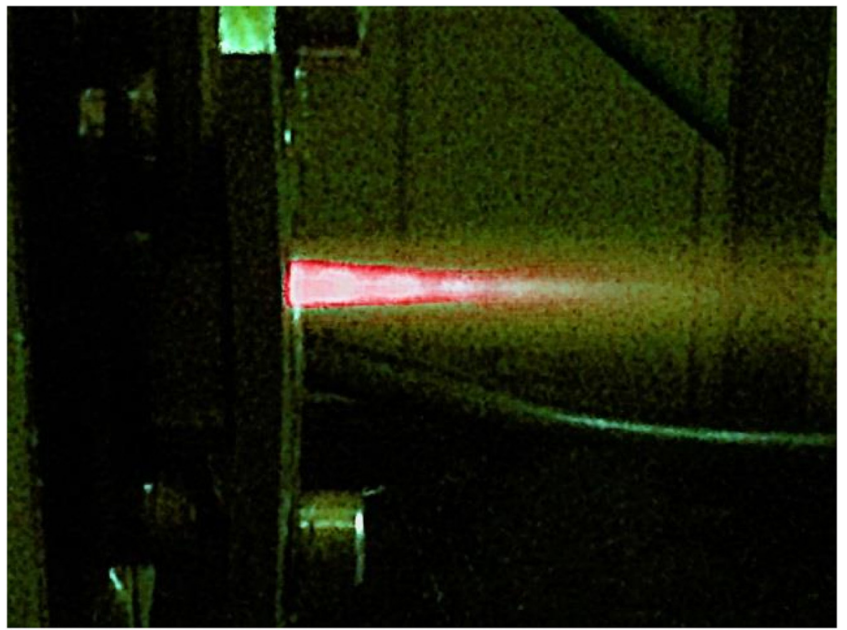

Fig. 4. Supersonic plasma jet.

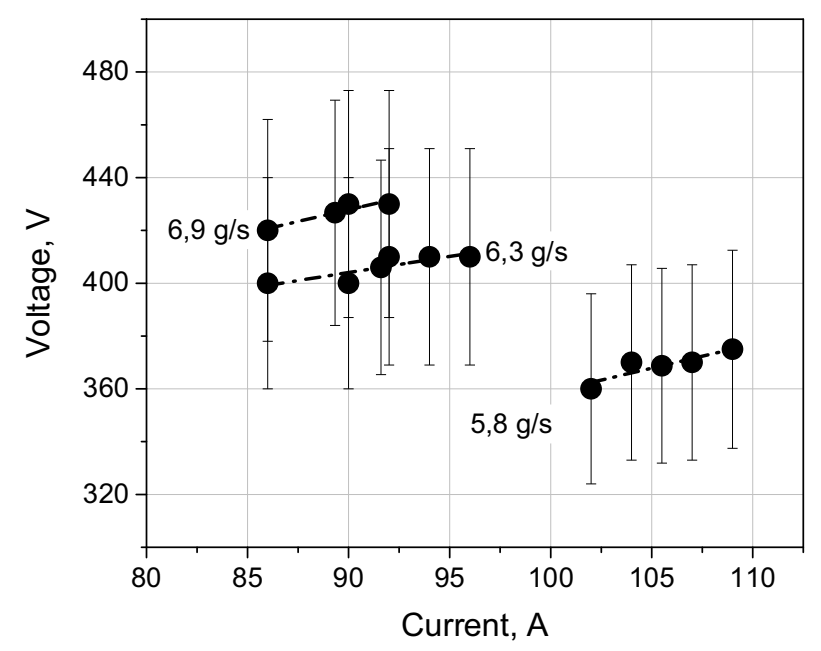

Fig. 5. Volt-Current characteristics of HVPS.

the atmospheric one, and the jet velocity, respectively, is reduced. Breaking supersonic flow naturally leads to the appearance of shock waves. If the excess pressure is sufficiently large, the jet velocity attains a critical value again, and after that supersonic one, i.e. a second supersonic expanded part appears. Due to losses in the first group of shock waves, the second overexpansion of the jet and the second group of shocks is weaker than the first. A similar picture is observed when the pressure in the plasma torch discharge chamber is lower than the designed one. In this case of the exhaust flow is compressed and pressure is increased. The compression may be so strong that the plasma jet pressure exceeds the atmospheric pressure. As a result, in both cases, appears a diamond-like structure, clearly observed on Fig. 4. For an ideal gas, this process of expansion and contraction would continue on forever creating an infinite number of Mach disks.

The current-voltage plot is the main electrical characteristic of a plasma torch, which describes the inter-relation between arc voltage and arc current under other fixed working parameters. In Fig. 5 the current-voltage characteristics of the plasma torch for supersonic spraying are shown. Voltage is almost independent on current and a slight increase (within the measurement accuracy) trend can be observed. U-shaped characteristic is typical when the arc length is fixed, as proposed by Zhukov and Zasypkin (2007). A reversed vortex, in addition to the fixed length of arc, stimulated a distributed blowing of cold gas into the arc, which has gas-dynamic

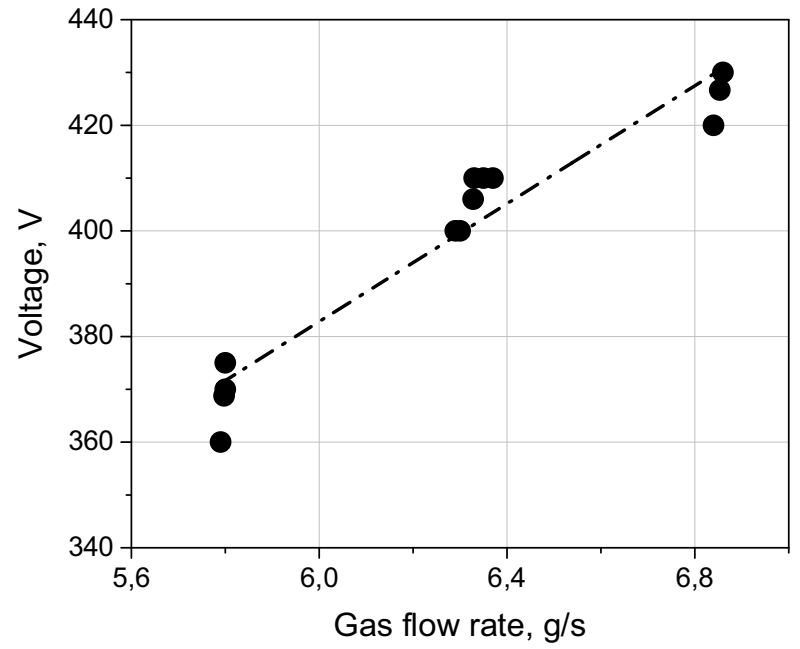

Fig. 6. Arc voltage vs gas flow rate.

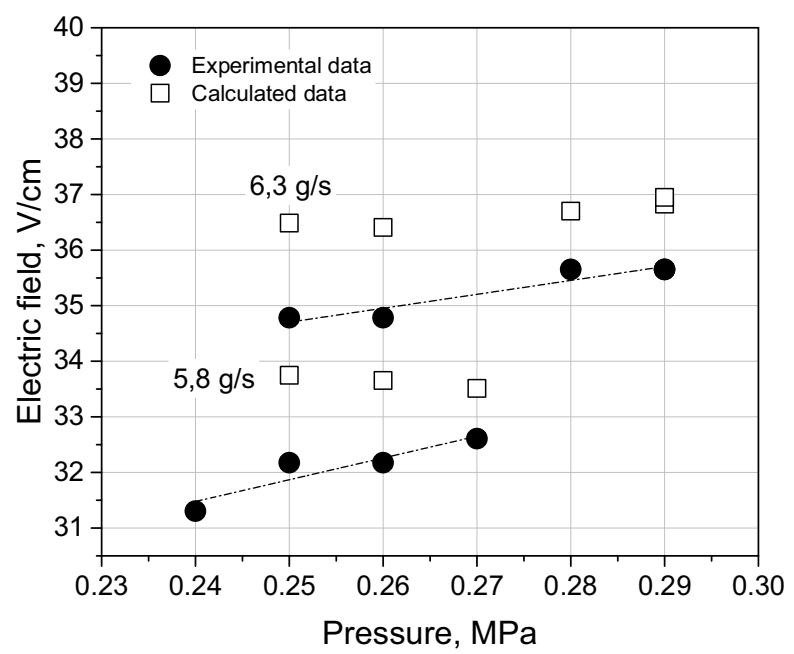

Fig. 7. Electric field strength vs pressure in discharge chamber.

effect on the strength of the electrical field, as shown experimentally by Essiptchouk et al. (2009). A developed turbulent flow may increase 2-3 times the electric field strength, as reported by Zhukov and Zasypkin (2007).

An outstanding feature of this plasma torch is the elevated voltage and low current, which is exactly the opposite of common plasma torches used for plasma spray (see Table 2). A low current ensures low erosion rate, low contamination of plasma and increase the lifetime of the electrodes. For the supersonic regime, the arc current was limited between $80 \mathrm{~A}$ and $110 \mathrm{~A}$.

The characteristic curves were obtained for three flow rates of nitrogen. The voltage dependence on the gas flow rate is shown in Fig. 6. Increase of flow rate in $20 \%$ promotes an increase of $15 \%$ of arc voltage due to intensification of heat exchange while the total pressure in the discharge chamber grows insignificantly.

An effective electric field $E=U / I$, where $U$ is total arc voltage and $l=$ constant is the inter-electrode distance, presented in Fig. 7, shows relatively high values that are typical for turbulent flow. It is observed that the electric field grows with increasing of the discharge chamber pressure. The experimental data of electric field (closed points) is compared with data from empirical depen- 


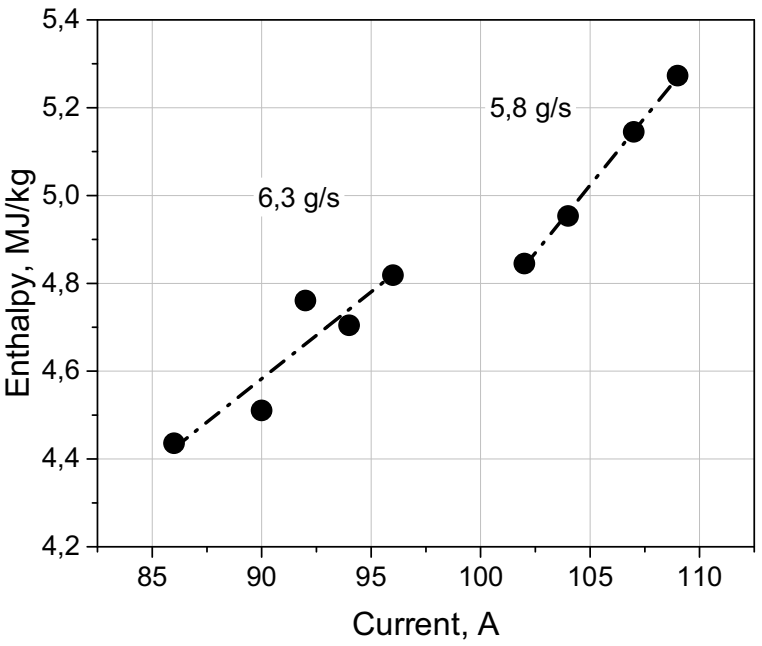

Fig. 8. Bulk enthalpy of plasma jet vs arc current.

dence (open points) obtained via Eq. (6) as proposed by Zhukov and Zasypkin (2007),

$E d=115\left(\frac{I}{d}\right)^{-0.23}\left(\frac{G_{N 2}}{d}\right)^{0.47}\left(p_{\text {torch }} d\right)^{0.2}$

where $d$ is the diameter of discharge chamber, $m$.

All this justify a practically linear increase of bulk enthalpy of plasma jet with arc current (Fig. 8). The plasma torch herein developed does not only produce a more stable jet but makes it also easier to adjust the enthalpy of the plasma gas by fine correction of the arc current.

The bulk enthalpy $\Delta h$ was estimated, as detailed on item 2.3. Thermal losses in the plasma torch were obtained from the measurement of the cooling water flow rate and its temperature. For temperature measurement the chromel-alumel thermocouples (type $\mathrm{K}$ ) were used. For the torch developed in this work the power ranged between 38 and $40 \mathrm{~kW}$, the efficiency of transformation electrical energy in thermal one was $75-80 \%$, which is higher than the values of the linear plasma torches, as calculated by Shanmugavelayutham and Selvarajan (2003) and Dorier et al., 2001.

Preliminary studies of kinetic and thermal characteristics of HVPS were carried out with application of an in-flight Particle Sensor DPV-2000. In-flight measurements for the metallic powder CoNiCrAlY (Fig. 9) represent the behaviour of the particles along the length of the supersonic plasma jet. The profile of particle velocity ranged between 425 and $500 \mathrm{~m} / \mathrm{s}$ (Fig. 9a), whereas the particle velocity starts to decrease at $150 \mathrm{~mm}$ from the nozzle. The particle temperature remained among the range of $2270-2440^{\circ} \mathrm{C}$. As observed by Fauchais (2004) the plasma spray process depends mainly on material precursor properties and transport (size, composition, specific mass, thermal conductivity, latent heat, method of fabrication, method of injection, etc) as well as plasma jet characteristics (working gas type and flow rate, torch design). From the interaction of previous parameters the respective particle residence time and heat transfer efficiency between particle-plasma will determine the range of particle velocity and temperature (Fauchais et al., 2013a,b). Within the works conducted to measure the in-flight CoNiCrAlY properties, the study of Richier et al. (2008) who deposited the CO-127 (CoNiCrAlY 5-45 $\mu \mathrm{m}$, from Praxair) by means of Cold Gas Dynamic Spraying - CGDS, reached a particle velocity of $560 \mathrm{~m} / \mathrm{s}$. Sampath et al. (2009) used the commercial plasma torch 7MB (Sulzer Metco), to spray the powder CO-211 (CoNiCrAlY, 5-90 $\mu \mathrm{m}$, from Praxair) at average particle velocitiy of $90 \mathrm{~m} / \mathrm{s}$. The data calculated by Yang et al. (2002) considered the properties of powder CO-210-1 (CoNiCrAlY, 10-45 $\mu \mathrm{m}$, from Praxair) with average grain size of $30 \mu \mathrm{m}$, using High Velocity Oxygen Fuel, could provide average velocity of $410 \mathrm{~m} / \mathrm{s}$ at a stand-off distance of $200 \mathrm{~mm}$. Comparison of velocities obtained on literature for different spray processes allows observe that the HVPS process, used in this work (see Fig. 9), can operate within the range of particle velocities of CGDS and the same range of $v_{p}$ and $T_{p}$ of HVOF process, which along with Vacuum Plasma Spray (VPS), are the state-of-art thermal spray technologies capable to produce high particle velocities and therefore high quality coatings.

A set of experiments were also performed in order to evaluate the influence of $\mathrm{G}_{\mathrm{N} 2}, \mathrm{G}_{\text {powder }}$ and stand-off distance on the particle velocity and temperature of $7 \%$ YSZ. According to Fig. 10, the particle velocity ranged between 491 and $683 \mathrm{~m} / \mathrm{s}$ and particle temperatures $2535-2636^{\circ} \mathrm{C}$. Thermal spray processes operating at high particle velocities may find limitation to deposit high melting point materials, due to the inherent low residence time. This trade-off becomes especially critical when processing ceramic materials. Conventional APS process, with radial injection, eventually encounters such limitations. The commonly adopted solution is to maximize the residence time by means, for example, of operation under quasi-laminar plasma flow, as described by Solonenko and Smirnov (2014) or increase of plasma enthalpy (Tarasi et al., 2008). The HVPS process shows, via in-flight particle velocity and temperature of 7\%YSZ (Fig. 10), that it is plausible to operate under high velocities/temperature with high melting point materials. The further analysis of 7\%YSZ in-flight particle data shows that the particle temperature (Fig. 10b) remained almost the same as commonly achieved from conventional APS process. Salimijazi et al. (2007) used a SG-100 (Praxair) plasma torch to plasma spray the Amperit 825 at $307 \mathrm{~m} / \mathrm{s}$ and $2836^{\circ} \mathrm{C}$. Chen et al. (2008) developed a high efficient plasma torch, working with a mixture of carbon dioxide and hydrocarbons and used it to spray the Amperit 825 at $205 \mathrm{~m} / \mathrm{s}$ and $2768^{\circ} \mathrm{C}$. When using the HVPS process developed in this work, the Amperit 825 reaches $491-683 \mathrm{~m} / \mathrm{s}$ and $2535-2636^{\circ} \mathrm{C}$ (Fig. 10). Despite the different set-up on the experiments of 7\%YSZ (runs 1 through 8 , see Table 2) the particle temperature was maintained almost constant, even with variation of the stand-off distance (between 100 and $150 \mathrm{~mm}$ ). This behavior can be atributed to the high efficiency heat transfer provided the plasma torch design, supersonic plasma jet regime and inherent efficient heat transfer environment provided by the gas nitrogen. Another important observation is that during 7\%YSZ in-flight analysis experiments, a narrow range of electric power input has been covered, which may have affected the results of particle temperature. Whilst, the $7 \%$ YSZ particle velocity showed a broader range. This may be sustained by the wide range of gradient pressure obtained during the experiments, the stand-off distance analysed and the low particle size.

The statistical analysis of particle velocity and temperature distribution obtained from single particle measurement from DPV-2000, provides sustainable discussion on particle state, as demonstrated by Streibl et al. (2006). In-flight data of CoNiCrAlY were analysed at different standoff distances by means of frequency distribution and a mono or multimodal fitting approximation has been adjusted, considering a Gauss distribution function. Statistical analysis of CoNiCrAlY data showed that $v_{p}$ distribution is monomodal until $100 \mathrm{~mm}$ (Fig. 11a), after that a bimodal distribution is observed (Fig. 11b). The average value decrease from 522

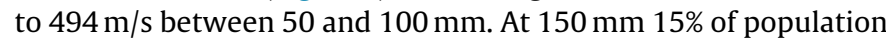
distribution assumes a $v_{p}$ of $387 \mathrm{~m} / \mathrm{s}$, and the remaining $519 \mathrm{~m} / \mathrm{s}$, whereas at $200 \mathrm{~mm} 37 \%$ of population distribution has an average $\mathrm{v}_{\mathrm{p}}$ of $393 \mathrm{~m} / \mathrm{s}$ and the rest $447 \mathrm{~m} / \mathrm{s}$. The $T_{\mathrm{p}}$ assumes a bimodal Gauss distribution, regardless the standoff distance analysed from the nozzle (Fig. 11c). For a standoff distance of $50 \mathrm{~mm}$ the $T_{p}$ frequency distribution is divided in a half, with average values of $2153^{\circ} \mathrm{C}$ and 

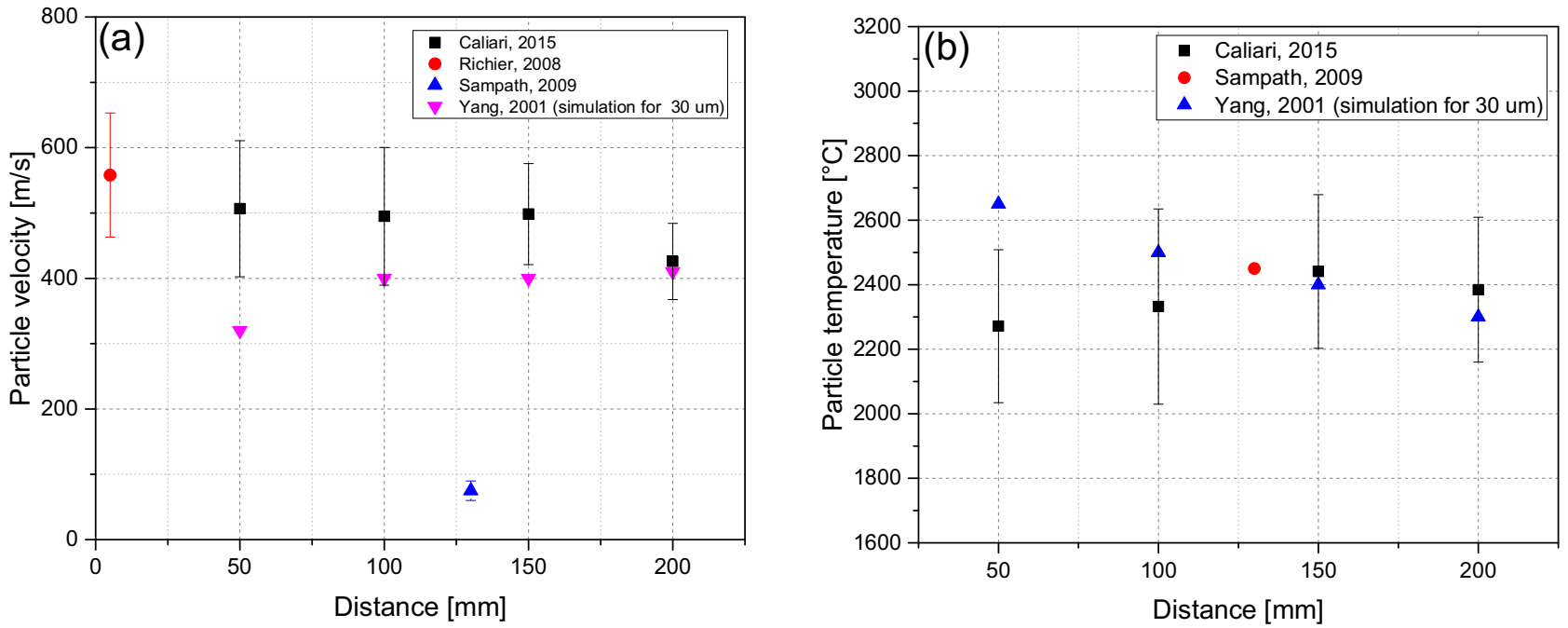

Fig. 9. Measurements of (a) velocity and (b) temperature of CoNiCrAlY particles on along the supersonic plasma jet.
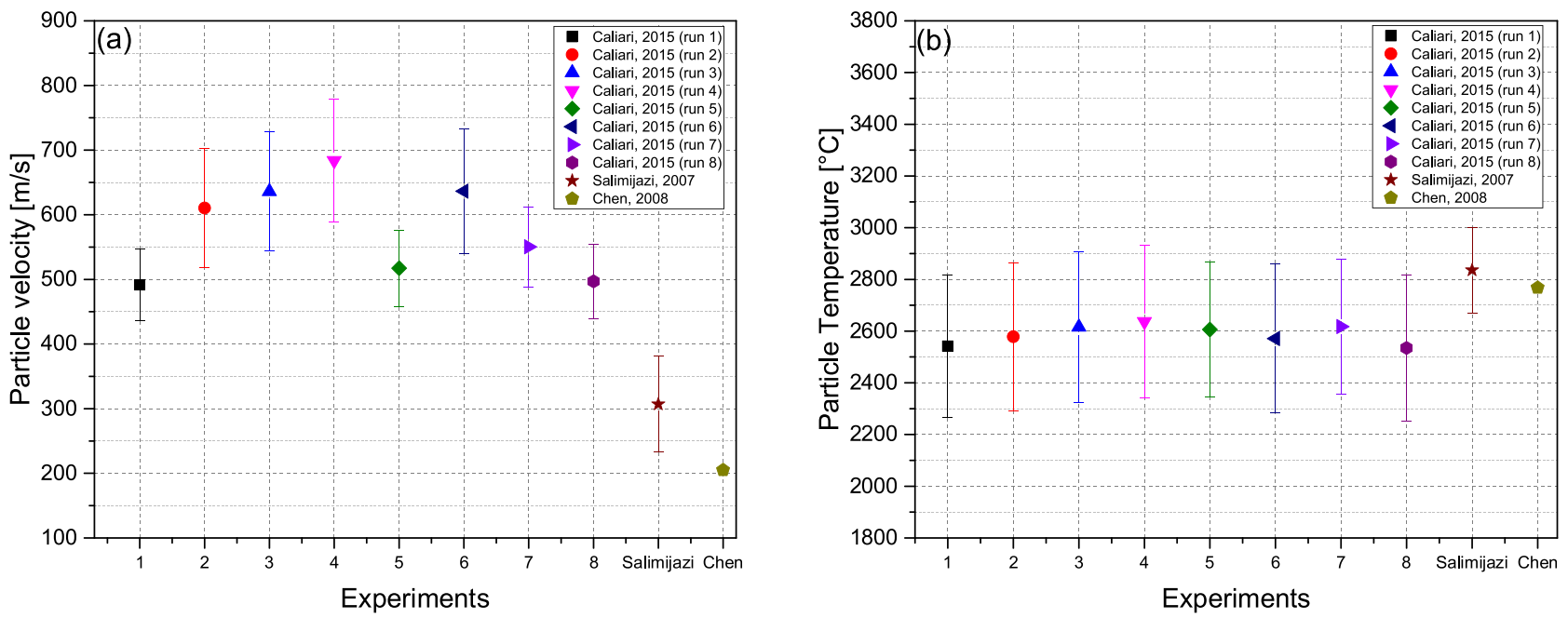

Fig. 10. Measurements of (a) velocity and (b) temperature of YSZ particles for different experiments.

$2427^{\circ} \mathrm{C}$. Between 100 and $150 \mathrm{~mm}$ the $38 \%$ of the particles present an increase in average value, from $2128^{\circ} \mathrm{C}$ to $2370^{\circ} \mathrm{C}$, whereas the remaining particles maintain around $2500^{\circ} \mathrm{C}$. The average values maintain at a distance of $200 \mathrm{~mm}$ further from the nozzle, nevertheless an increase to $52 \%$ of the population with the lowest average value of particle temperature $\left(2370^{\circ} \mathrm{C}\right)$ is observed.

The analysis of particle properties distribution is critical when processing high melting point materials, such as ceramics, because it may compromise coating quality, as well as deposition efficiency. For instance, Mauer et al. (2009) studied the correlation of plasma spray parameters with the corresponding coating porosity and deposition efficiency, during the deposition of YSZ. The results indicated that high deposition efficiency and low porosity is achieved at increasing values of particle temperature, which presented an increase of the dispersion of particle temperature distribution. Conventionally, high melting particles injected radially to plasma jet exhibit a multimodal and highly dispersive distribution of temperature, as described by Sampath et al. (2009). The histograms of run 3 represented on Fig. 12 allows to demonstrate that both $v_{p}$ and $T_{p}$ distributions assume a monomodal-Gaussian profile on the HVPS process.

Other important parameter of plasma spraying is fluctuation of arc voltage together with the power spectrum of arc voltage. Con- sidering small arc length variations, it is expected that HVPS has low current and voltage variation and, respectively, low enthalpy and plasma jet temperature fluctuations. A typical picture of arc voltage fluctuation is shown in Fig. 13a, and the power spectrum of voltage (Fig. 13b). The data was obtained by Digital Storage Oscilloscope Tektronix TDS2024C (200 MHz, 4-Ch, 2 GS/s). Besides chaotic low amplitude pulsations (low-scale shunting) it is clearly observed a large-scale oscillation of arc voltage. For the typical discharge of the average arc voltage of $370 \mathrm{~V}$ a standard deviation of voltage fluctuation of $\pm 10 \%$ was obtained. According to Fauchais (2004), for conventional plasma torches the voltage fluctuations vary between $\pm 15 \%$ and $\pm 35 \%$. The power spectrum on frequency was analyzed by means of Fast Fourier Transform (FFT), which was gathered at a sampling rate of $25 \mathrm{kS} / \mathrm{s}$. The spectrum of voltage on the frequency domain was adjusted using a low pass filtering. According to Fig. 13b the spectrum does not indicated a characteristic frequency peak, except the initial peak related to the power supply.

One interesting phenomenon was observed during supersonic plasma spraying. Some particles directed out of spraying axis, were forced to merge back into the plasma jet, as shown in Fig. 14. That behaviour can be explained by the Magnus effect, which determines the force acting on a rotating body in flow. The particles start to rotate when crossing a high velocity gradient in the supersonic jet 

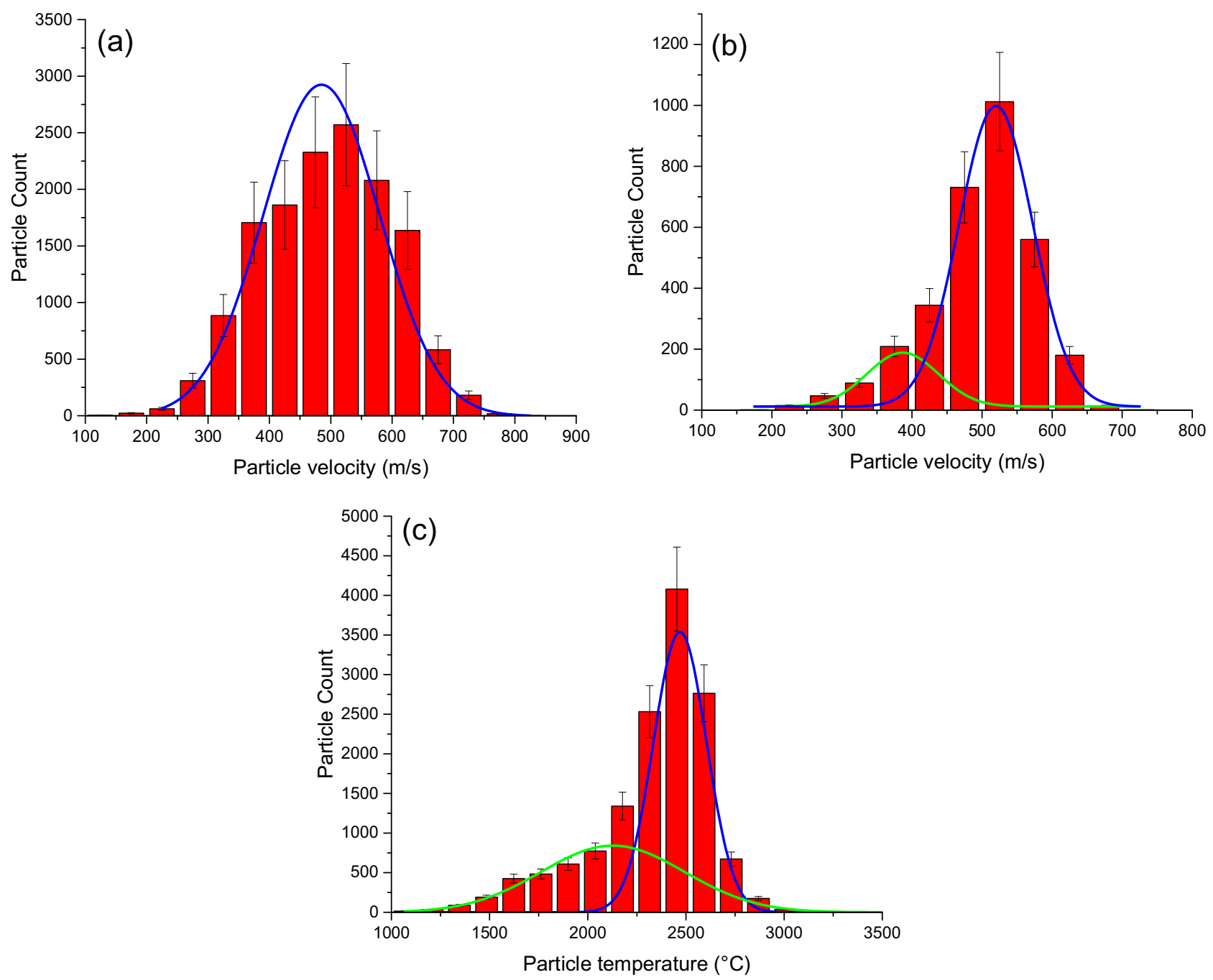

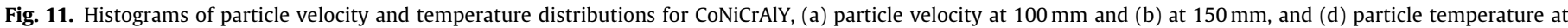
$100 \mathrm{~mm}$.
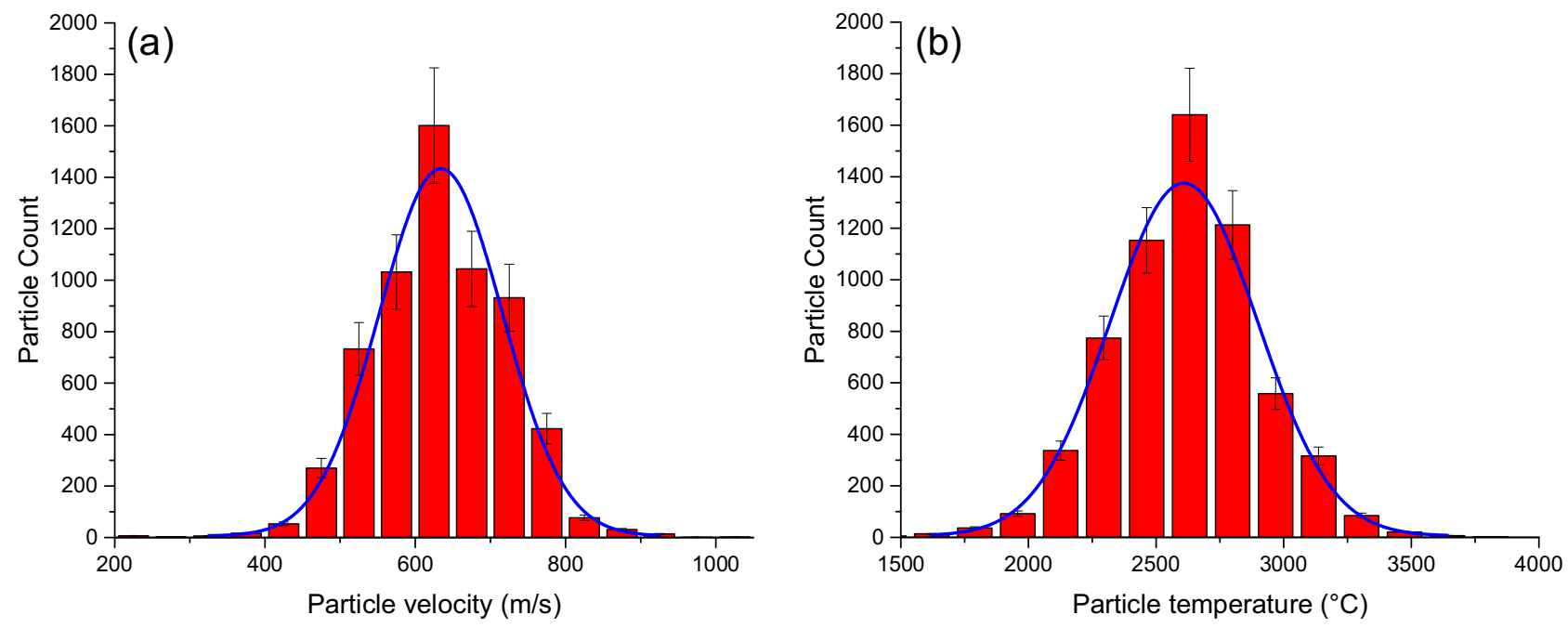

Fig. 12. Histograms of particle velocity and temperature distributions for $7 \%$ YSZ particle of run 3 .

boundary layer (shock wave). This rotation creates a pressure gradient on particle surface directed to the jet axis. The acting force is proportional to the upstream velocity and the vortex strength established by particle rotation. The force direction can be obtained from the vector product $\vec{v} \times \vec{\omega}$, between the plasma flow velocity $\vec{v}$ and the particle angular velocity $\vec{\omega}$. 

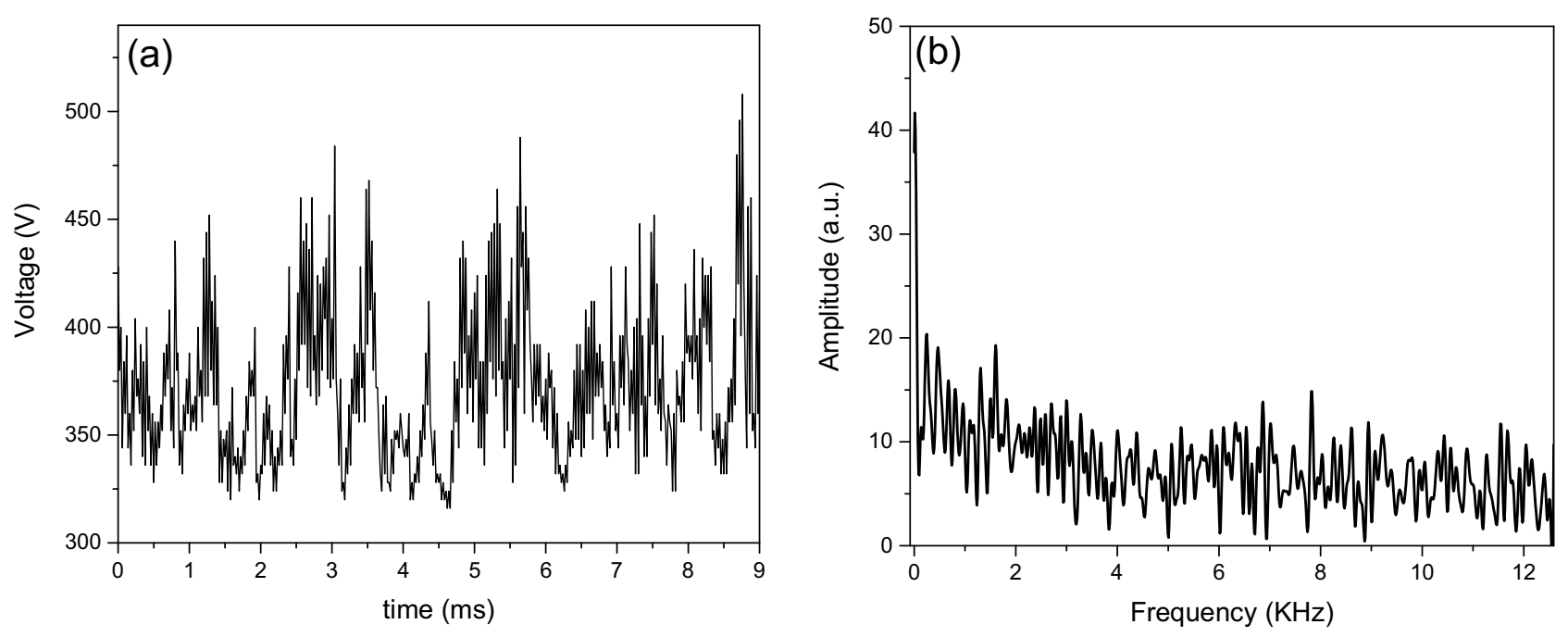

Fig. 13. (a) Arc voltage fluctuation and (b) power spectrum.

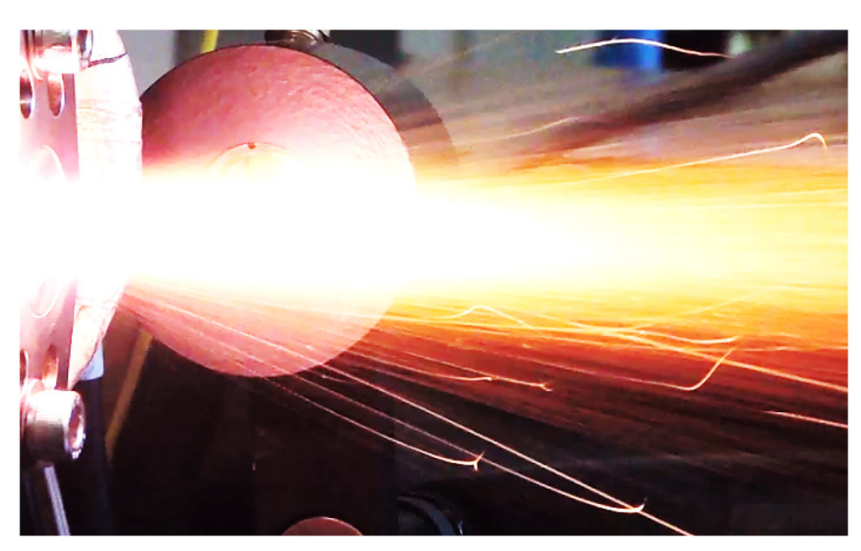

Fig. 14. Sprayed particle flow.

\section{Conclusions}

A plasma torch design with discharge chamber axis perpendicular to feedstock injection direction has been discussed. The current voltage characteristics has slightly ascending trend, with a fixed arc length. Electrical, thermal and kinetic characteristics outlined from comparison with conventional linear plasma spray torches are intermediate between APS, HVOF and VPS. The plasma torch developed in this work showed an elevated arc voltage $(370 \mathrm{~V})$ and low arc current $(100 \mathrm{~A})$, which contribute to increase the electrode life and decrease the arc voltage relative fluctuation (10\%). The results of in-flight analysis using DPV-2000 showed that CoNiCrAlY particles were sprayed at velocities of $500 \mathrm{~m} / \mathrm{s}$ and temperature of $2400{ }^{\circ} \mathrm{C}$. The ceramic $7 \% \mathrm{YSZ}$ were sprayed at $491-683 \mathrm{~m} / \mathrm{s}$ and $2535-2636^{\circ} \mathrm{C}$. Histogram of particle velocity and temperature shows a monomodal and bimodal distributions. The presented plasma torch works with overall thermal efficiency of 75-80\% and provides an elevated heat transfer efficiency considering the plasma-particle interaction.

\section{Acknowledgments}

The authors acknowledge the financial support grant \#2012/24851-7 provided by São Paulo Research Foundation (FAPESP), CNPq and CAPES of Brazil.

\section{References}

Caliari, F.R., Miranda, F.S., Reis, D.A.P., Filho, G.P., Charakhovski, L.I., Essiptchouk, A., 2015. New kind of plasma torch for supersonic coatings at atmospheric pressure. In: Proceedings of the 22nd International Symposium on Plasma Chemistry, July 510, Antwerp, Belgium, 6 p.

Chen, L., Pershin, L., Mostaghimi, J., 2008. A new highly efficient high-power DC plasma torch. IEEE Trans. Plasma Sci. 36 (4), 1068-1069.

Chyou, Y.P., Pfender, E., 1989. Behaviour of particulates in thermal plasma flows, Plasma Chem. Plasma Process. 9, 45-71.

DPV-2000 reference manual., 2009. Tecnar Rev. 5, 44.

Dorier, J.L., Hollenstein, C., Salito, A., Loch, M., Barbezat, G., 2001. Characterization and origin of arc fluctuations in a F4 DC plasma torch used for thermal spraying, High Temp. Material Processes., 5, 477-489.

Essiptchouk, A.M., Charakhovski, L.I., Filho, G.P., Maciel, H.S., Otani, C., Barros, E.A., 2009. Thermal and power characteristics of plasma torch with reverse vortex. J. Phys. D: Appl. Phys. 42, 6.

Fauchais, P., 2004. Understanding plasma spraying. J. Phys. D: Appl. Phys. 37, R86-R108.

Fauchais, P., Heberlein, J., Boulos, M.I., 2013a. Thermal Spray Fundamentals From Powder to Part, XXVIII. Springer, US, pp. 953.

Fauchais, P., Montavon, G., 2007. Plasma spraying: from plasma generation to coating structure. Adv. Heat Transfer 40, 205-344.

Fauchais, P., Montavon, G., Lima, R.S., Marple, B.R., 2011. Engineering a new class of thermal spray nano-based microstructures from agglomerated nanostructured particles, suspensions and solutions: an invited review. J. Phys. D: Appl. Phys. 44, 53.

Fauchais, P., Vardelle, M., Vardelle, A., 2013b. Reliability of plasma-sprayed coatings: monitoring the plasma spray process and improving the quality of coatings. J. Phys. D: Appl. Phys. 46, 16.

Hermanek, F.J., 2001. Thermal Spray Terminology and Company Origins. ASM International, Materials Park, $\mathrm{OH}$.

Mauer, G., Vassen, R., Stöver, D., 2009. Atmospheric plasma spraying of yttria-stabilized zirconia coatings with specific porosity. Surf. Coat. Technol. 204, 172-179.

Mohanty, P., Stanisic, Jovan, Stanisic, Jelena, George, A., Wang, Y.A., 2010. Study on arc instability phenomena of an axial injection cathode plasma torch. J. Therm. Spray Technol. 19, 465-475.

Nogues, E., Vardelle, M., Fauchais, P., Granger, P., 2008. Arc voltage fluctuations: comparison between two plasma torch types. Surf. Coat. Technol. 202, 4387-4393.

Papyrin, A., Kosarev, V., Klinkov, S., Alkhimov, A., Fomin, V., 2006. Cold Spray Technology, 1st edition. Elsevier Science, 328p.

Pawlowski, L., 2008. The Science and Engineering of Thermal Spray Coatings, 2nd ed. John Wiley \& Sons Ltd, 626p.

Richier, P., Zúñiga, A., Yandouzi, M., Jodoin, B., 2008. CoNiCrAlY microstructural changes induced during cold gas dynamic spraying. Surface \& Coatings Technology 203, 364-371.

Sampath, S., Srinivasan, V., Valarezo, A., Vaidya, A., Streibl, T., 2009. Sensing, control, and In situ measurement of coating properties: an integrated approach toward establishing process-Property correlations. J. Therm. Spray Technol. 18 (2), 243-255.

Shanmugavelayutham, G., Selvarajan, V., 2003. Electrothermal efficiency, temperature and thermal conductivity of plasma jet in a DC plasma spray torch. PRAMANA- J. Phys. Indian Acad. Sci. 61, 1109.

Salimijazi, H.R., Pershin, L., Coyle, T.W., Mostaghimi, J., Chandra, S., Lau, Y.C., Rosenzweig, L., Moran, E., 2007. Effect of droplet characteristics and substrate 
surface topography onthe final morphology of plasma-sprayed zirconia single splats. J. Thermal Spray Technol. 16 (2), 291-299.

Solonenko, O.P., Smirnov, A.V., 2014. Advanced oxide powders processing based on cascade plasma. 13th high-tech plasma processes conference (HTPP 2014). J. Phys. Conf. Ser. 550

Streibl, T., Vaidya, A., Friis, M., Srinivasan, V., Sampath, S., 2006. A critical assessment of particle temperature distributions during plasma spraying: experimental results for YSZ. Plasma Chem. Plasma Process. 26 (1), 73-102.

Tarasi, F., Medraj, M., Dolatabadi, A., Oberste-Berghaus, J., Moreau, C., 2008. Effective parameters in axial injection suspension plasma spray process of alumina-zirconia ceramics. J. Therm. Spray Technol. 17 (5-6), 685-691.

Van Steenkiste, T.H., Smith, J.R., Teets, R.E., Moleski, J.J., Gorkiewicz, D.W., Tison, R.P., Marantz, D.R., Kowalsky, K.A., Riggs, W.L., Zajchowski, P.H., Pilsner, B., McCune, R.C., Barnett, K.J., 1999. Kinetic spray coatings. Surf. Coat. Technol. $111,62-71$.
Vardelle, A., Moreau, C., Themelis, N.J., Chazelas, C., 2015. A perspective on plasma spray technology. Plasma Chem. Plasma Process. 35, 491-509.

Vardelle, M., Vardelle, A., Fauchais, P., Saray, I., 1994. Comparison of classical and axial injection torches for spraying alumina coatings. Mater. Manuf. Processes 9, 735-755.

Yang, Y.-M., Liao, H., Coddet, C., 2002. Simulation and application of a HVOF process for MCrAlY thermal spraying. J. Therm. Spray Technol. 11 (1), 36-43.

Zhukov, M.F., Zasypkin, I.M., 2007. Thermal Plasma Torches-Design, Characteristics, Applications, 1st ed. Cambridge International Science Publishing Ltd, 596p. 